\title{
VARIABLE POWER SINGULAR INTERFACE ELEMENTS FOR A CRACK NORMAL TO THE BIMATERIAL INTERFACE
}

\author{
WU YONG-LI \\ Institute of Mechanics, Chinese Academy of Sciences, Beijing 100080, P.R.C.
}

\begin{abstract}
Ahstract-Zero thickness crack tip interface elements for a crack normal to the interface between two materials are presented. The elements are shown to have the desired $r^{-1}(0<\lambda<1)$ singularity in the stress field at the crack tip and are compatible with other singular elements. The stiffness matrices of the quadratic and cubic interface element are derived. Numerical examples are given to demonstrate the applicability of the proposed interface elements for a crack perpendicular to the bimaterial interface.
\end{abstract}

\section{INTRODUCTION}

As THE DEMANDS made on the strength and durability of today's engineering materials increase, the advantages of combining the properties of two or more materials into a single member have become apparent. However, this same combination of material properties can contribute to failure of these structures. Clearly, the integrity of the junction between the materials is of particular interest. When the failure of such a structure involves the cracking or debonding of bimaterial interface, fracture mechanics provides an excellent method of studying the problem. As the complex geometries and loading of many crack problems of homogeneous and composite materials do not lend themselves to analytical solutions, the finite element method has gained acceptance as a possible solution tool for these problems. Most finite element models that are used currently in the analysis of the fracture mechanics incorporate special singular finite elements that model the singular stress conditions at the crack tip. The quadratic quarter point singular finite elements developed by Henshell and Shaw [1] and Barsoum [2] are the commonly used singular crack tip elements. These elements have the theoretical $r^{-1 / 2}$ singularity and therefore aid in the efficient modeling of the stresses near the tip of a crack.

A successful finite element should be able to model the properties of the interface between the two materials in a composite material, as the properties of the interface can have a significant effect on the strength and the fracture toughness of the composite. Goodman et al. [3] proposed zero width linear or quadratic bond interface elements to model the interface region of the composite. Tarazi and Mandel [4] developed a special five noded, quadratic, zero width, singular interface finite element for modeling the interface of the fiber and the matrix material at the crack tip of an interface crack in a composite material. The element has the required theoretical $r^{-1 / 2}$ singularity in its stress field. In this paper special quadratic and cubic zero width singular interface finite elements are developed for modeling a crack normal to the bimaterial interface. These elements have the desired theoretical $r^{i-1}$ singularity where $\lambda(0<\lambda<1)$ is the lowest root of the characteristic equation depending on the elastic constants of two materials [5]. These singular interface elements can be used easily and efficiently with the collapsed isoparametric elements which have also the variable power singularity of the stress field at the crack tip [6]. Numerical examples are given to demonstrate the applicability of the proposed interface elements for modeling a crack normal to an interface of bimaterials. The results have also been used to display the effect of the interface shear and tensile stiffness on the fracture parameter and deformation of composite materials.

\section{QUADRATIC INTERFACE ELEMENT}

Consider the quadratic interface element shown as Fig. 1. Using the quadratic shape functions $N_{t}$, the element geometry in the local coordinate system $x, y$ can be mapped into the one-dimensional normalized space:

$$
x=\sum_{i=1}^{3} N_{i} x_{i}=\sum_{i=1}^{3} N_{i+3} x_{i+3},
$$


where

$$
\begin{aligned}
& N_{1}=N_{6}=\zeta(\zeta-1) / 2, \\
& N_{2}=N_{5}=\left(1-\zeta^{2}\right), \\
& N_{3}=N_{4}=\zeta(1+\zeta) / 2 .
\end{aligned}
$$

Furthermore the displacements of the elements in the local coordinate system $x, y$ can be expressed as:

$$
\begin{aligned}
& u_{\mathrm{u}}=\sum_{i=1}^{3} N_{t+3} u_{t+3}, \quad v_{\mathrm{u}}=\sum_{i=1}^{3} N_{t+3} v_{i+3}, \\
& u_{1}=\sum_{i=1}^{3} N_{i} u_{i}, \quad v_{1}=\sum_{i=1}^{3} N_{i} v_{1} .
\end{aligned}
$$

$u_{\mathrm{u}}$ and $v_{\mathrm{u}}$ refer to the $x$ and $y$ displacements of the upper side of the element (side $4,5,6$ ), while $u_{1}$ and $v_{1}$ refer to displacements of the lower side of the element (side 1,2,3). The relative displacements of the upper and lower sides can be written as

$$
\left\{\begin{array}{l}
u \\
v
\end{array}\right\}=\left[\begin{array}{cccccccccccc}
-N_{1} & 0 & -N_{2} & 0 & -N_{3} & 0 & N_{4} & 0 & N_{5} & 0 & N_{6} & 0 \\
0 & -N_{1} & 0 & -N_{2} & 0 & -N_{3} & 0 & N_{4} & 0 & N_{5} & 0 & N_{6}
\end{array}\right]\left\{\begin{array}{c}
u_{1} \\
v_{1} \\
\vdots \\
u_{6} \\
v_{6}
\end{array}\right\}
$$

or

$$
\left\{\begin{array}{l}
u \\
v
\end{array}\right\}=[N]\left\{\begin{array}{l}
u_{i} \\
v_{i}
\end{array}\right\}
$$

In a first approximation the interfacial zone is represented by one-dimensional extensional and shear springs distributed over a surface. For linear behavior in extension and shear the conditions across the interface may then be expressed as

and

$$
\{P\}=\left\{\begin{array}{l}
p_{x} \\
p_{y}
\end{array}\right\}=[K]\left\{\begin{array}{l}
u \\
v
\end{array}\right\}
$$

$$
[K]=\left[\begin{array}{cc}
k_{\mathrm{r}} & 0 \\
0 & k_{y}
\end{array}\right]
$$

where $k_{x}$ and $k_{y}$ are suitable values for the bond stiffness per unit area between the materials in the $x$ and $y$ direction, respectively.

It should be noted that eq. (6) includes the case of perfect contact $\left(k_{x}=k_{y}=\infty\right)$, when the stree and displacements are continuous, and the case of no contact $\left(k_{r}=k_{y}=0\right)$ when the stresses vanish.

Using eq. (6) the work done during the deformation of the element can be expressed as

$$
W=\frac{1}{2} \int_{\mathrm{r}_{1}}^{x_{2}}\left\{\begin{array}{l}
u \\
v
\end{array}\right\}\{P\} \mathrm{d} x=\frac{1}{2} \int_{\mathrm{r}_{1}}^{x_{2}}\left\{\begin{array}{l}
u \\
v
\end{array}\right\}^{\mathrm{T}}[K]\left\{\begin{array}{l}
u \\
v
\end{array}\right\} \mathrm{d} x .
$$

From eq. (1) we have

$$
\mathrm{d} x=\frac{1}{2}\left(L_{1} \zeta+L\right) \mathrm{d} \zeta
$$

where

$$
\begin{aligned}
L_{1} & =2\left(x_{1}+x_{3}-2 x_{2}\right), \\
L & =x_{3}-x_{1} .
\end{aligned}
$$

Substituting eqs (5) and (9) into eq. (8), we get

$$
W=\frac{1}{2}\left\{\begin{array}{l}
u_{i} \\
v_{i}
\end{array}\right\}^{\top}\left[K_{v y}\right]\left\{\begin{array}{l}
u_{i} \\
v_{l}
\end{array}\right\}
$$


where $\left[K_{r y}\right]$ is the stiffness matrix of the interface elements.

$\left[K_{\mathrm{ry}}\right]=$

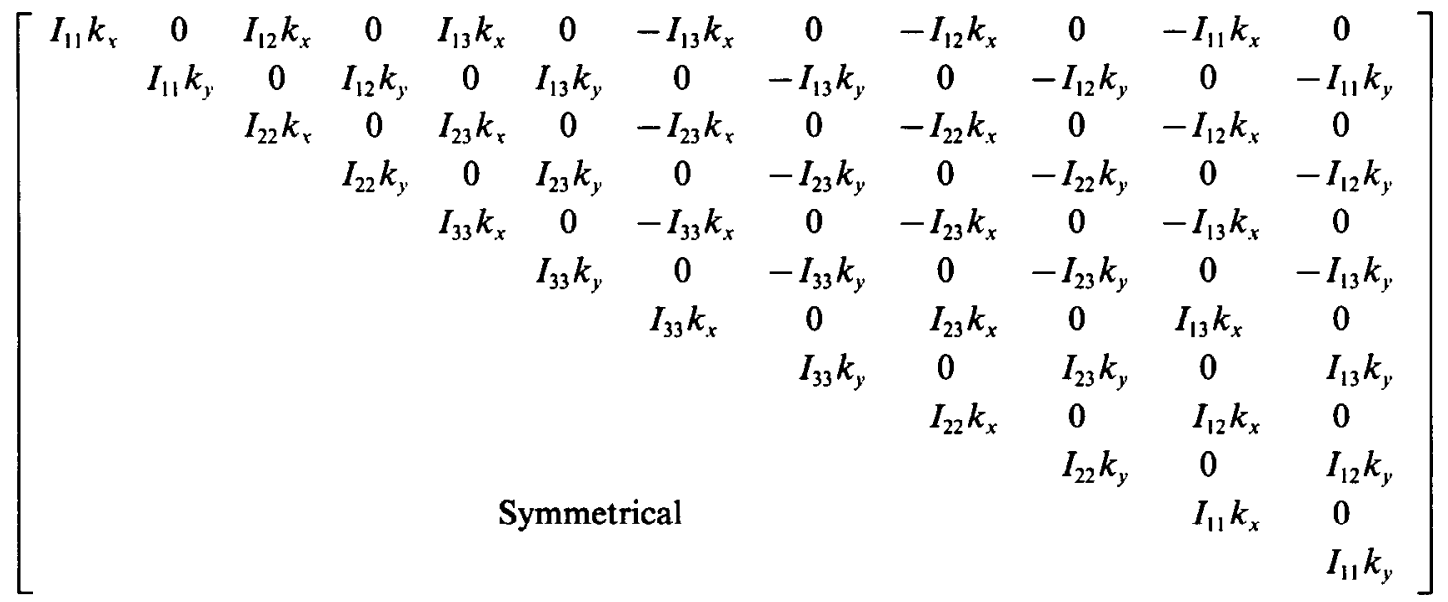

where

$$
\begin{array}{ll}
I_{11}=\frac{1}{2} \int_{-1}^{1} N_{1}^{2}\left(L+L_{1} \zeta\right) \mathrm{d} \zeta=\frac{1}{10}\left(\frac{4}{3} L-L_{1}\right), & I_{22}=\frac{1}{2} \int_{-1}^{1} N_{2}^{2}\left(L+L_{1} \zeta\right) \mathrm{d} \zeta=\frac{8}{15} L, \\
I_{33}=\frac{1}{2} \int_{-1}^{1} N_{3}^{2}\left(L+L_{1} \zeta\right) \mathrm{d} \zeta=\frac{1}{10}\left(\frac{4}{3} L+L_{1}\right), & I_{12}=\frac{1}{2} \int_{-1}^{1} N_{1} N_{2}\left(L+L_{1} \zeta\right) \mathrm{d} \zeta=\frac{1}{15}\left(L-L_{1}\right), \\
I_{13}=\frac{1}{2} \int_{-1}^{1} N_{1} N_{3}\left(L+L_{1} \zeta\right) \mathrm{d} \zeta=-\frac{1}{30} L, & I_{23}=\frac{1}{2} \int_{-1}^{1} N_{2} N_{3}\left(L+L_{1} \zeta\right) \mathrm{d} \zeta=\frac{1}{15}\left(L+L_{1}\right) .
\end{array}
$$

It can show that the interface clement has the desired strain singularity by choosing adequately the location of the side-node.

Without loss of generality consider the lower side of the element and assume

$$
x_{1}=0, \quad x_{2}=\alpha x_{3} .
$$

From eq. (10) $L_{1}$ and $L$ can be expressed as

$$
L_{1}=2 x_{3}(1-2 \alpha), \quad L=x_{3} .
$$

Substituting eqs (2) and (14) into eq. (1) we get

$$
x=x_{3}\left[\left(\frac{1}{2}-\alpha\right) \zeta^{2}+\frac{1}{2} \zeta+\alpha\right] .
$$

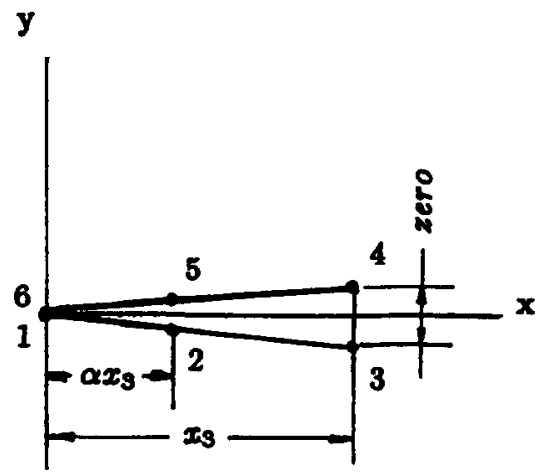

Fig. 1. The quadratic singular interface element.

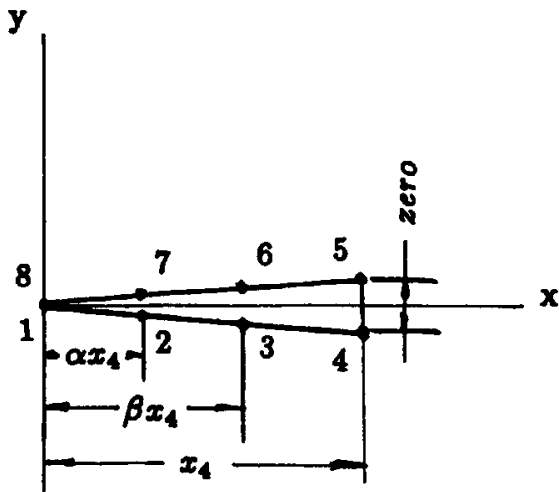

Fig. 2. The cubic singular interface element. 
The least-squares method can be used to determine $\alpha$ values for various values of $\lambda$ by satisfying the condition [7]

$$
\left(\frac{1}{2}-\alpha\right) \zeta^{2}+\frac{1}{2} \zeta+\alpha=\left(\frac{1+\zeta}{2}\right)^{1 /,}, \text { for }-1 \leqslant \zeta \leqslant 1
$$

Equation (16) can be written as

$$
x=x_{3}\left(\frac{1+\zeta}{2}\right)^{1 / k}
$$

From eqs (3) and (18) we get

$$
\frac{\partial u}{\partial x}=\frac{\partial u}{\partial \zeta} \frac{\partial \zeta}{\partial x}=4\left(u_{1}-2 u_{2}+u_{3}\right)\left(\frac{\lambda}{x_{3}}\right)\left(\frac{x}{x_{3}}\right)^{2 .}-\left(3 u_{1}-4 u_{2}+u_{3}\right)\left(\frac{\lambda}{x_{3}}\right)\left(\frac{x}{x_{3}}\right)^{i-1}
$$

It is seen that the strain has $r^{2 i-1}$ and $r^{i-1}$ singularities. The term $r^{2 \lambda-1}$ is the nonsingularity for $2 \lambda-1 \geqslant 0$. For $\lambda=1$ this is the case for a usual interface element which has no singularity. In the case $\lambda=1 / 2$, we get $\alpha=1 / 4$ and $L_{1}=L$ from eqs (17) and (10). The strains have singularity which is the case considered by Tarazi and Mandel [4]. Therefore eqs (12) and (13) can be used with the usual and variable power singular interface elements.

With the above matrix formulations all that remains is to transform the matrix in the local coordinate system $x, y$ to the stiffness matrix in the global coordinate system $x^{\prime}, y^{\prime}$. This can be done through the following transformation [7]

$$
\left[K_{x^{\prime} y^{\prime}}\right]=\left[\begin{array}{cc}
{[S]^{\mathrm{T}}} & 0 \\
0 & {[S]^{\mathrm{T}}}
\end{array}\right]\left[K_{x y}\right]\left[\begin{array}{cc}
{[S]} & 0 \\
0 & {[S]}
\end{array}\right]
$$

The angle $\theta$ is the angle between the local coordinate system $x, y$ and the global coordinate system $x^{\prime}, y^{\prime}$.

\section{CUBIC INTERFACE ELEMENT}

Consider now the cubic interface element as shown in Fig. 2.

$$
\begin{gathered}
x=\sum_{i=1}^{4} N_{i} x_{i}=\sum_{i=1}^{4} N_{i+4} x_{i+4} \\
u_{\mathrm{u}}-\sum_{i=1}^{4} N_{t+4} u_{i+4}, \quad v_{\mathrm{u}}=\sum_{i=1}^{4} N_{i+4} v_{t+4} \\
u_{1}=\sum_{i=1}^{4} N_{i} u_{i}, \quad v_{1}=\sum_{i=1}^{4} N_{i} v_{l}
\end{gathered}
$$

where

$$
\begin{aligned}
& N_{1}=N_{8}=(1-\zeta)\left(9 \zeta^{2}-1\right) / 16 \\
& N_{2}=N_{7}=9(1-3 \zeta)\left(1-\zeta^{2}\right) / 16 \\
& N_{3}=N_{6}=9(1+3 \zeta)\left(1-\zeta^{2}\right) / 16 \\
& N_{4}=N_{5}=(1+\zeta)\left(9 \zeta^{2}-1\right) / 16
\end{aligned}
$$

From eqs (21) and (24) we get

$$
\mathrm{d} x=\left(L+L_{1 \zeta}+L_{2 \zeta}^{2}\right) \mathrm{d} \zeta
$$

where

$$
\begin{aligned}
L & =\left(x_{1}-27 x_{2}+27 x_{3}+x_{4}\right) / 16 \\
L_{1} & =9\left(x_{1}-x_{2}-x_{3}+x_{4}\right) / 8 \\
L_{2} & =27\left(-x_{1}+3 x_{2}-3 x_{3}+x_{4}\right) / 16 .
\end{aligned}
$$

Following the procedure outlined before, the stiffness matrix of the cubic interface element can be obtained. 
Modeling element behavior at the bimaterial interface

767

정

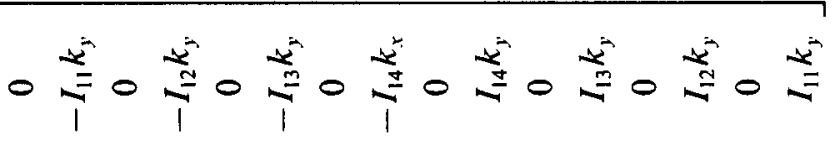

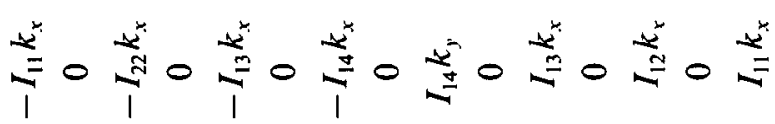

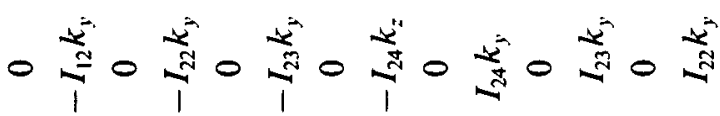

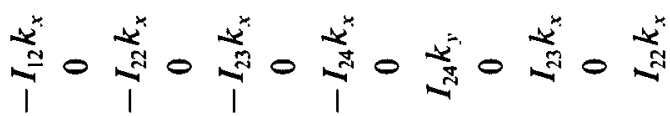

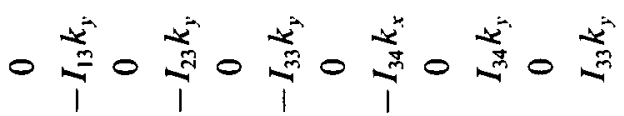

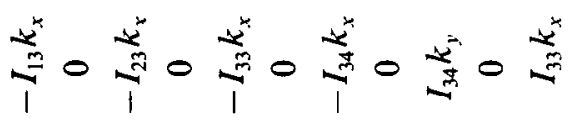

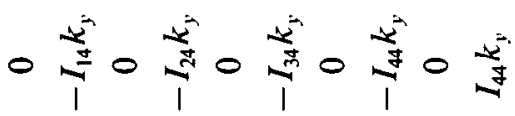

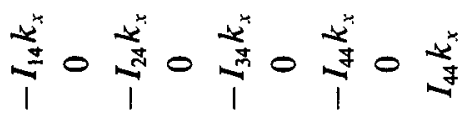

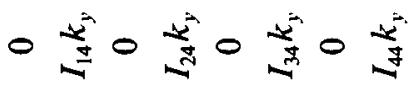

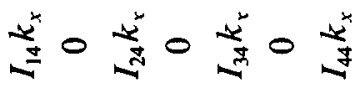

$$
\begin{aligned}
& 0=0
\end{aligned}
$$

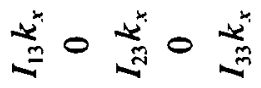

$$
\begin{aligned}
& 0 \text { ०ै है }
\end{aligned}
$$

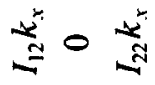

$$
\begin{aligned}
& 0
\end{aligned}
$$

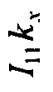


where

$$
\begin{aligned}
& I_{11}=\int_{-1}^{1} N_{1}^{2}\left(L+L_{1} \zeta+L_{2} \zeta^{2}\right) \mathrm{d} \zeta=\frac{1}{840}\left(128 L-109 L_{1}+98 L_{2}\right) \text {, } \\
& I_{22}=\int_{-1}^{1} N_{2}^{2}\left(L+L_{1} \zeta+L_{2} \zeta^{2}\right) \mathrm{d} \zeta=\frac{27}{280}\left(8 L-3 L_{1}-2 L_{2}\right) \\
& I_{33}=\int_{-1}^{1} N_{3}^{2}\left(L+L_{1} \zeta+L_{2} \zeta^{2}\right) \mathrm{d} \zeta=\frac{27}{280}\left(8 L+3 L_{1}-2 L_{2}\right), \\
& I_{44}=\int_{-1}^{1} N_{4}^{2}\left(L+L_{1} \zeta+L_{2} \zeta^{2}\right) \mathrm{d} \zeta=\frac{1}{840}\left(128 L+109 L_{1}+98 L_{2}\right) \\
& I_{12}=\int_{-1}^{1} N_{1} N_{2}\left(L_{1}+L_{1} \zeta+L_{2} \zeta^{2}\right) \mathrm{d} \zeta=\frac{3}{280}\left(11 L-10 L_{1}+7 L_{2}\right) \\
& I_{13}=\int_{-1}^{1} N_{1} N_{3}\left(L+L_{1} \zeta+L_{2} \zeta^{2}\right) \mathrm{d} \zeta=\frac{3}{280}\left(-4 L+5 L_{1}-2 L_{2}\right), \\
& I_{14}=\int_{-1}^{1} N_{1} N_{4}\left(L+L_{1} \zeta+L_{2} \zeta^{2}\right) \mathrm{d} \zeta=\frac{1}{840}\left(19 L+11 L_{2}\right) \\
& I_{23}=\int_{-1}^{1} N_{2} N_{3}\left(L+L_{1} \zeta+L_{2} \zeta^{2}\right) \mathrm{d} \zeta=-\frac{27}{280}\left(L+L_{2}\right) \\
& I_{24}=\int_{-1}^{1} N_{2} N_{4}\left(L+L_{1} \zeta+L_{2} \zeta^{2}\right) \mathrm{d} \zeta=-\frac{1}{280}\left(4 L+5 L_{1}+2 L_{2}\right) \text {, } \\
& I_{34}=\int_{-1}^{1} N_{3} N_{4}\left(L+L_{1} \zeta+L_{2} \zeta^{2}\right) \mathrm{d} \zeta=\frac{3}{280}\left(11 L+10 L_{1}+7 L_{2}\right) .
\end{aligned}
$$

Consider the lower side of element and assume

$$
x_{1}=0, \quad x_{2}=\alpha x_{4}, \quad x_{3}=\beta x_{4} .
$$

Substituting eqs (24) and (29) into eq. (21) we obtain

$$
x=\frac{x^{4}}{16}\left[9(1+3 \alpha-3 \beta) \zeta^{3}+9(1-\alpha-\beta) \zeta^{2}+(27 \beta-27 \alpha-1) \zeta+(9 \alpha+9 \beta-1)\right] .
$$

The least-squares method associated with a simplex method can be used to determine $\alpha$ and $\beta$ values for different $\lambda$ values by satisfying the condition [7]

$$
\begin{aligned}
\frac{9}{16}(1+3 \alpha-3 \beta) \zeta^{3}+\frac{9}{16}(1-\alpha-\beta) \zeta^{2}+\frac{1}{16}(27 \beta-27 \alpha-1) \zeta+\frac{1}{16}(9 \alpha+9 \beta-1) & =\left(\frac{1+\zeta}{2}\right)^{1 / i}, \text { for }-1 \leqslant \zeta \leqslant 1
\end{aligned}
$$

Equation (30) can be written

$$
x=x_{4}\left(\frac{1+\zeta}{2}\right)^{1 / \lambda}
$$

In that case we obtain

$$
\frac{\partial u}{\partial x}=A_{1}\left(\frac{x}{x_{4}}\right)^{i-1}+A_{2}\left(\frac{x}{x_{4}}\right)^{2 i-1}+A_{3}\left(\frac{x}{x_{4}}\right)^{3 x-1},
$$




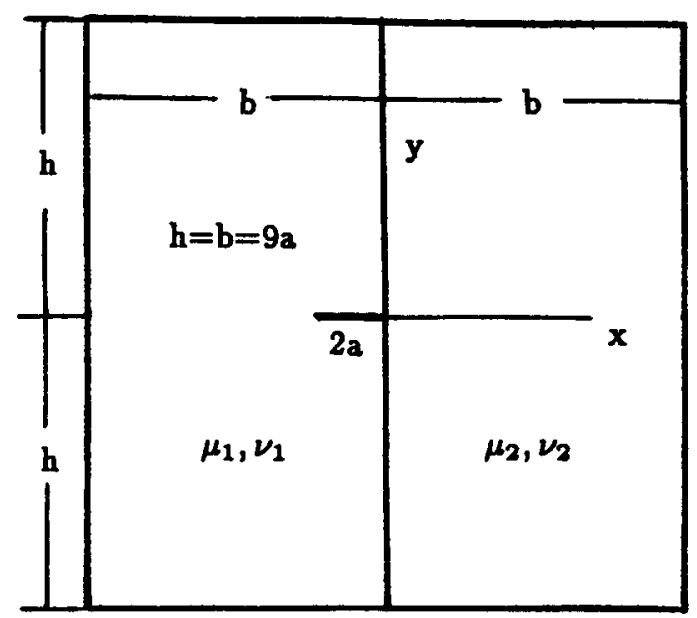

Fig. 3. Cracked bimaterial strip.

where

$$
\begin{aligned}
& A_{1}=\frac{1}{4}\left(-22 u_{1}-45 u_{2}+63 u_{3}+4 u_{4}\right) \frac{\lambda}{x_{4}}, \\
& A_{2}=\left(18 u_{1}-45 u_{2}+36 u_{3}-9 u_{4}\right) \frac{\lambda}{x_{4}}, \\
& A_{3}=\frac{1}{2}\left(-27 u_{1}+81 u_{2}-81 u_{3}+27 u_{4}\right) \frac{\lambda}{x_{4}} .
\end{aligned}
$$

Equation (33) above shows that the strain of the cubic interface element has $r^{i-1}, r^{2 i-1}, r^{3 i-1}$ singularities. The terms $r^{3 i-1}$ and $r^{2 i-1}$ are the nonsingularity for $2 \lambda-1 \geqslant 0$. The term $r^{3 i-1}$ is the nonsingularity for $3 \lambda-1 \geqslant 0$. For the case $\lambda=1$ we have $\alpha=1 / 3, \beta=2 / 3, L_{1}=0$ and $L_{2}=0$ from eqs (31), (29) and (26). It is the usual interface element which has no singularity. For the interface crack the eigenvalue is $1 / 2$. It can show

$$
\begin{aligned}
& \alpha=1 / 9, \quad \beta=4 / 9 \\
& L=x_{4} / 2, \quad L_{1}=3 x_{4} / 2, \quad L_{2}=0 .
\end{aligned}
$$

This cubic singular interface element is compatible with the cubic singular isoparametric element [6].

\section{NUMERICAL EXAMPLE}

Consider a bimaterial strip with a crack normal to and terminating at the interface as shown in Fig. 3. The crack surfaces are subjected to uniform pressure. Because of the symmetry of the geometry and load along the $x$-axis only half the model was analyzed. The finite element mesh

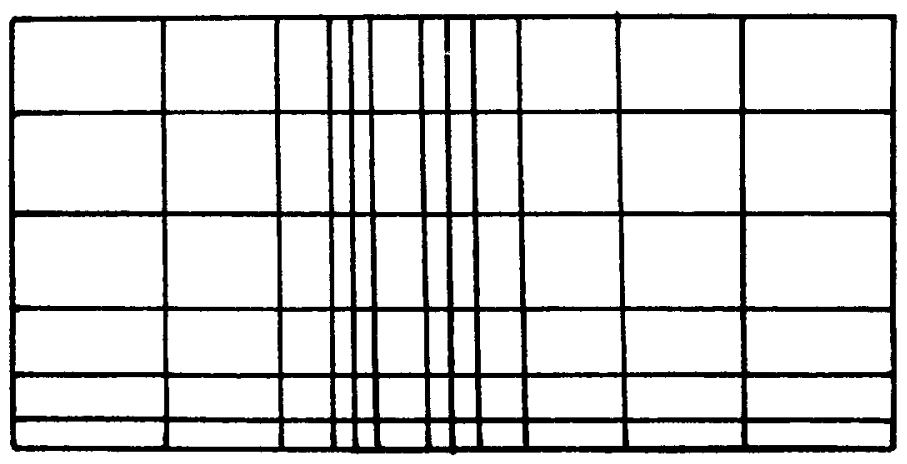

Fig. 4. Finite element mesh. 


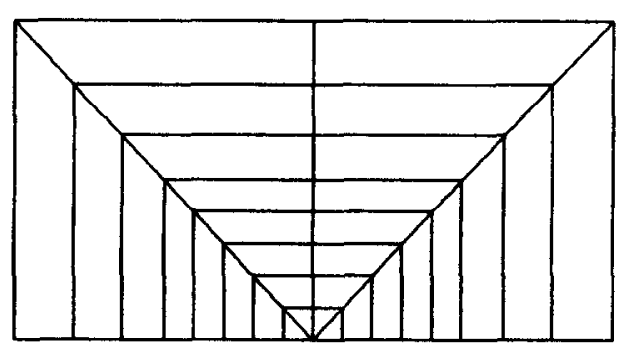

Fig. 5. Mesh in crack location.

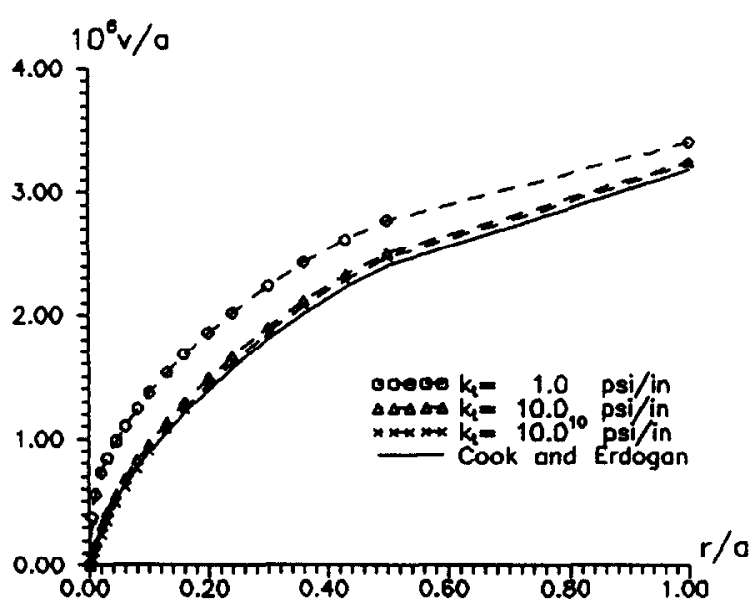

Fig. 6. Crack surface displacement $\left(k_{\mathrm{n}}=1.0 \mathrm{psi} / \mathrm{in}\right.$. $)$.

is shown in Figs 4 and 5. The total mesh involved 479 nodes, 132 quadratic isoparametric elements and 13 quadratic interface elements along the bimaterial interface. The present singular interface element was placed at the crack tip along the interface. The collapsed singular elements [6] modeled the remainder of crack tip region. The numerical calculation was carried for epoxy-aluminium with the following properties

$$
\begin{aligned}
& \mu_{1}=0.1667 \times 10^{6} \mathrm{psi}, \quad v_{1}=0.35, \\
& \mu_{2}=3.846 \times 10^{6} \mathrm{psi}, \quad v_{2}=0.3 .
\end{aligned}
$$

The eigenvalue $\lambda$ of the strain singularity for the material combination is 0.6619 . In order to obtain the desired strain singularity $r^{\lambda-1}$ for singular interface element and collapsed singular elements at the crack tip we have $\alpha=0.35021$ [7].

The finite element analysis was conducted for various values of tension stiffness $k_{\mathrm{n}}$ and shear stiffness $k_{\mathrm{t}}$. Figures 6,7 and 8 show the crack surface displacements for $k_{\mathrm{n}}=1.0,10.0,10^{10} \mathrm{psi} / \mathrm{in}$, respectively. The result of the singular integral equation obtained by Cook and Erdogan [8] for the perfect bonded interface was also shown. It can be seen from the results, as the stiffness $k_{\mathrm{n}}$ and $k_{\mathrm{t}}$ increases the crack surface displacements decrease and approach Cook's result. The present result for $k_{\mathrm{n}}=k_{\mathrm{t}}=10^{10} \mathrm{psi} / \mathrm{in}$. agree well with the result of the singular integral equation [8].

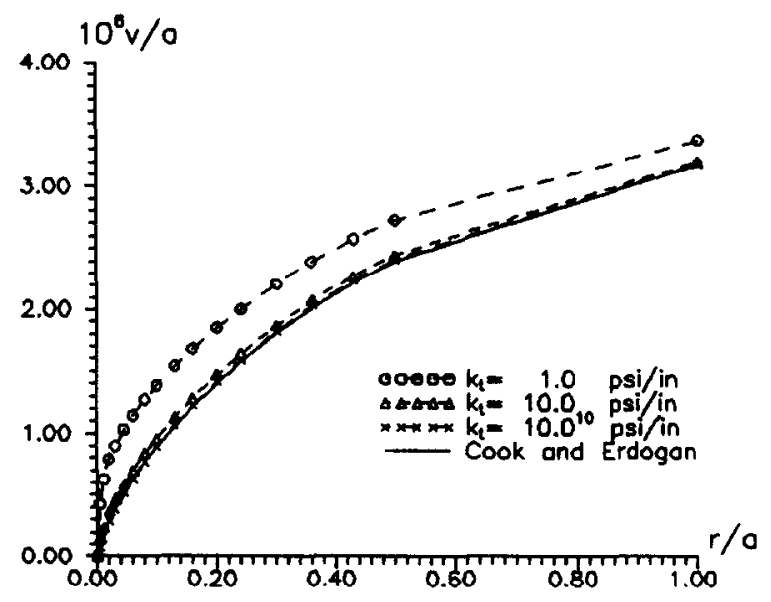

Fig. 7. Crack surface displacement $\left(k_{\mathrm{n}}=10.0 \mathrm{psi} / \mathrm{in}\right.$. $)$. 


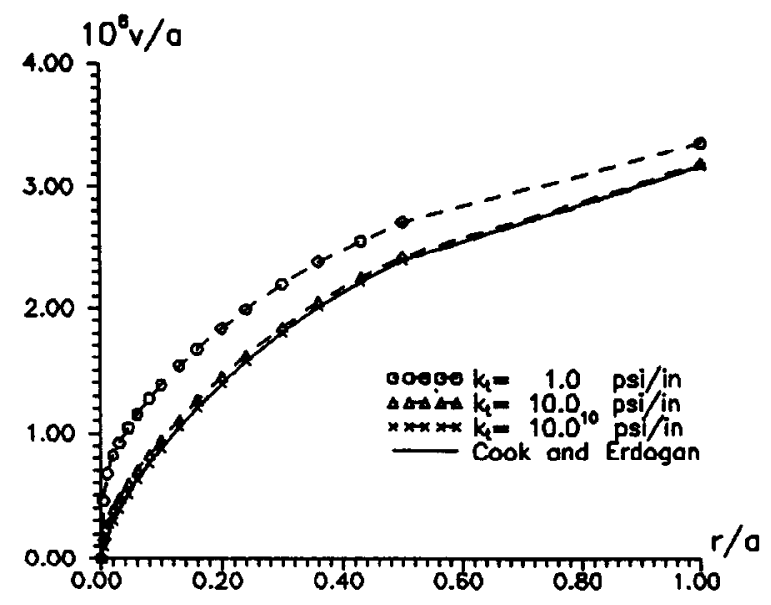

Fig. 8. Crack surface displacement $\left(k_{\mathrm{n}}=10.0^{10} \mathrm{psi} / \mathrm{in}\right.$.). as [8]

The stress intensity factor generalized for both the embedded and bondline crack tip is defined

$$
K=\sqrt{2 \pi} \lim _{r \rightarrow 0} r^{1-i} \sigma_{y y}(r, 0)
$$

To deduce $K$ from the displacement data the following equation was used

$$
K=\sqrt{2 \pi} \mu * u_{y}(r, \pi) / r^{i}
$$

where

$$
\begin{aligned}
\mu^{*} & =\mu_{2} \frac{(1-2 \lambda)\left(m+k_{2}\right)+(1+2 \lambda)\left(1+m k_{1}\right)}{\left(m+k_{2}\right)\left(1+m k_{1}\right) \sin \pi \lambda} \\
m & =\mu_{2} / \mu_{1} .
\end{aligned}
$$

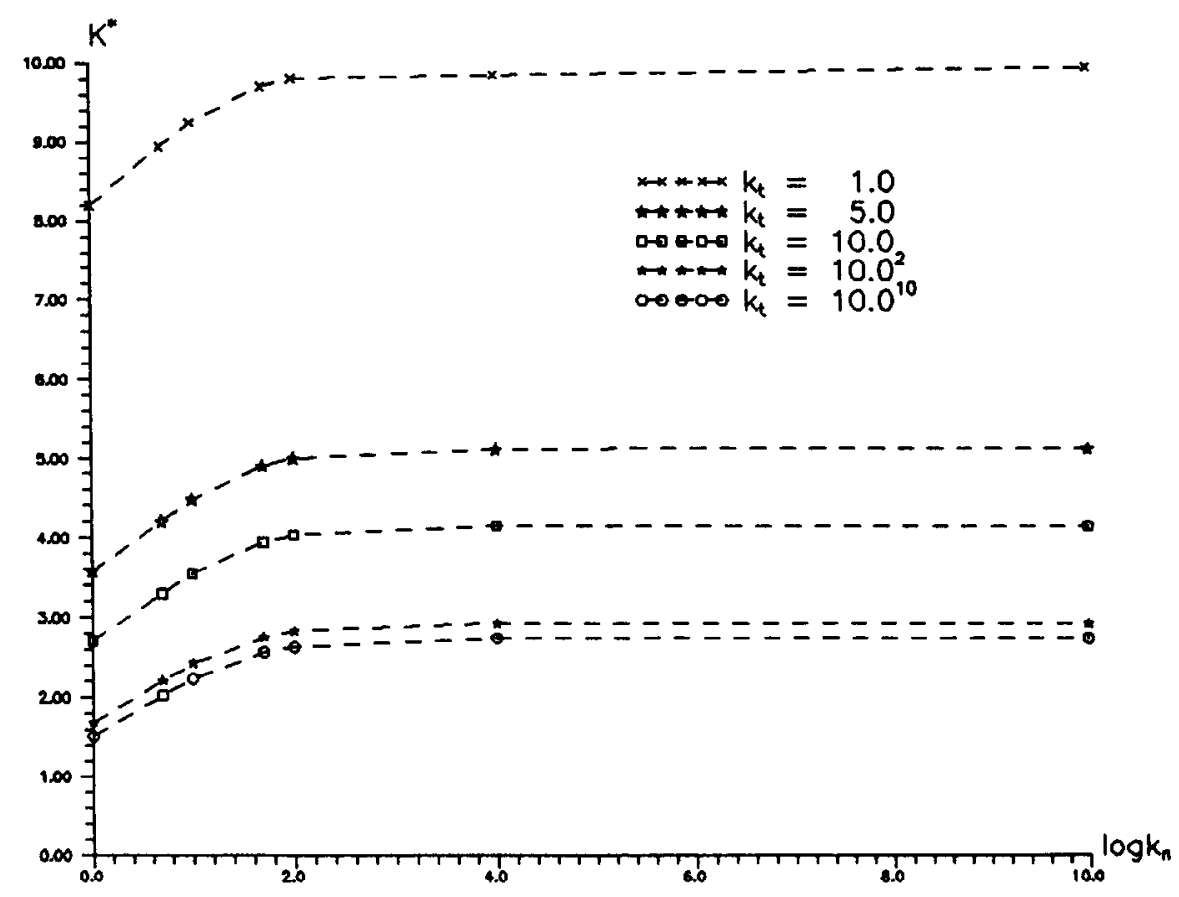

Fig. 9. $K^{*}$ vs $\log k_{\mathrm{n}}$ curves (unit for $k_{\mathrm{n}}$ and $k_{\mathrm{t}}: \mathrm{psi} / \mathrm{in}$.). 
From eq. (38) the normalized stress intensity factor $K^{*}=K / \sigma \sqrt{\pi} a^{1-\prime}$ at the bondline crack ends from the finite element data at $r / a=0.001$ can be found. Figure 9 shows curves of the $K^{*}$ vs $k_{\mathrm{n}}$ for various values $k_{\mathrm{t}}$. The value $K$ for $k_{\mathrm{n}}=k_{\mathrm{t}}=10^{10} \mathrm{psi} / \mathrm{in}$. was found to be 2.752 which agrees well with 2.7845 computed by use of the singular integral equation for a perfect bonded interface [8]. It is interesting to note that each curve in Fig. 9 is similar to the curve in Fig. 12 given by Tarazi and Mandel for the interface crack [4]. The stress intensity factor increases as tension stiffness $k_{\mathrm{n}}$ increases. But the stress intensity factor increases more rapidly as shear stiffness $k_{\mathrm{t}}$ decreases.

\section{CONCLUDING REMARKS}

The quadratic and cubic interface element can have the desired strain singularity by choosing adequately the location of the side-node. The stiffness matrix of the element is derived. The elements are shown to be compatible with the other singular isoparametric elements. The numerical results show that the element adequately models the deformations near the crack tip for a perpendicular interface crack. The numerical results also show that the tension stiffness and shear stiffness of the interface have different effects on the stress intensity factor.

Acknowledgements - This work was supported by National Natural Science Foundation of China and Laboratory for Nonlinear Mechanics of Continuous Media, Institute of Mechanics, Chinese Academy of Sciences.

\section{REFERENCES}

[1] R. D. Henshell and K. G. Shaw, Crack tip finite elements are unnecessary. Int. J. numer. Meth. Engng 9, 495-507 (1975).

[2] R. S. Barsoum, Triangular quarter-point element as elastic and perfectly-plastic crack lip elements. Int. J, numer. Meth. Engng 11, 85-98 (1977).

[3] R. E. Goodman. R. L. Taylor and T. L. Brekke, A model for the mechanics of jointed rock. J. Soil Mech. Foundation Division Proc. ASCE, pp. 636-659 (May 1968).

[4] S. S. Tarazi and J. Mandel, Zero thickness quarter point crack tip finite element for modeling an interface between two materials. Engng Fracture Mech. 30, 37-48 (1988).

[5] A. R. Zak and M. I. Williams, Crack point stress singularities at a bi-materials interface. J. appl. Mech. 30, 142-143 (1963).

[6] Wu Yong-li, The collapsed isoparametric elements as a singular element for a crack normal to the bimaterial interface. Comput. Structures, in press.

[7] R. El Abdi and G. Valentin, Isoparametric elements for crack normal to the interface between two bonded layers. Comput. Structures 33, 241-248 (1989).

[8] T. S. Cook and F. E. Erdogan, Stresses in bonded materials with a crack perpendicular to the interface. Int. J. Engng Sci. 10, 677-697 (1972). 